\title{
Interval estimation of trigonometrical splines
}

\author{
I.G. Burova ${ }^{1, *}$, E.G. Ivanova ${ }^{1}$, and V.A. Kostin ${ }^{1}$ \\ ${ }^{1}$ St. Petersburg State University, 7/9 Universitetskaya nab., \\ St.Petersburg, 199034 Russia
}

\begin{abstract}
Quite often, it is necessary to quickly determine variation range of the function. If the function values are known at some points, then it is easy to construct the local spline approximation of this function and use the interval analysis rules. As a result, we get the area within which the approximation of this function changes. It is necessary to take into account the approximation error when studying the obtained area of change of function approximation. Thus, we get the range of changing the function with the approximation error. This paper discusses the features of using polynomial and trigonometrical splines of the third order approximation to determine the upper and lower boundaries of the area (domain) in which the values of the approximation are contained. Theorems of approximation by these local trigonometric and polynomial splines are formulated. The values of the constants in the estimates of the errors of approximation by the trigonometrical and polynomial splines are given. It is shown that these constants cannot be reduced. An algorithm for constructing the variation domain of the approximation of the function is described. The results of the numerical experiments are given.
\end{abstract}

\section{Introduction}

It is useful to determine the lower and upper bounds of the values of functions, eigenvalues of operators, solutions of systems of linear and nonlinear equations without without calculating a detailed numerical solution of the corresponding problems. The solution of such problems is considered in many papers published recently.

In the paper [1] a new approach for solving non linear systems of equations was proposed. This approach is based on Interval- Newton and Interval-Krawczyk operators and $\mathrm{B}$-splines. The proposed algorithm is making great benefits of the geometric properties of B-spline functions to avoid unnecessary computations. For eigenvalue problems of self-adjoint differential operators, a universal framework is proposed to give explicit lower and upper bounds for the eigenvalues (see [2]).

To improve the calculation accuracy and reduce the computational cost, the interval analysis technique and radial point interpolation method are adopted in [3] to obtain the approximate frequency response characteristics for each focal element, and the corresponding formulations of structural-acoustic system for interval response analysis are deduced.

This paper continues the series of papers on approximation by local polynomial and non-polynomial splines and interval estimation see [5], [6],[7].

For constructing this interval extension, we use techniques from interval analysis.

\section{Approximation with the left splines}

Suppose $a, b$ be real numbers. Let the set of nodes $X_{j}$ be such that $a<\ldots<X_{j-1}<X_{j}<X_{j+1}<\ldots<b$.

\subsection{Trigonometric splines}

First, we consider the approximation of a function $f(x)$ by the left trigonometric splines (see [6]). We construct an approximation $F(x)$ of function $f(x), f \in C^{(3)}[a, b]$, $x \in\left[X_{j}, X_{j+1}\right]$ in the form:

$$
F(x)=f\left(X_{j-1}\right) w_{j-1}(x)+f\left(X_{j}\right) w_{j}(x)+f\left(X_{j+1}\right) w_{j+1}(x) .
$$

We obtain basic functions $w_{j-1}(x), w_{j}(x), w_{j+1}(x), x \in$ $\left[X_{j}, X_{j+1}\right]$, solving the following system:

$$
\begin{gathered}
w_{j-1}(x)+w_{j}(x)+w_{j+1}(x)=1 . \\
\sin \left(X_{j-1}\right) w_{j-1}(x)+\sin \left(X_{j}\right) w_{j}(x)+\sin \left(X_{j+1}\right) w_{j+1}(x)=\sin (x), \\
\cos \left(X_{j-1}\right) w_{j-1}(x)+\cos \left(X_{j}\right) w_{j}(x)+\cos \left(X_{j+1}\right) w_{j+1}(x)=\cos (x),
\end{gathered}
$$

The solution of this system can be written as follows:

$$
\begin{aligned}
& w_{j}(x)=\left(\sin \left(X_{j+1}-x\right)-\sin \left(X_{j+1}-X_{j-1}\right)+\sin \left(x-X_{j-1}\right)\right) / Z_{j}, \\
& w_{j+1}(x)=\left(-\sin \left(-x+X_{j}\right)+\sin \left(X_{j}-X_{j-1}\right)-\sin \left(x-X_{j-1}\right)\right) / Z_{j}, \\
& w_{j-1}(x)=\left(\sin \left(-x+X_{j}\right)-\sin \left(-X_{j+1}+X_{j}\right)-\sin \left(X_{j+1}-x\right)\right) / Z_{j},
\end{aligned}
$$

where $Z_{j}=\sin \left(X_{j}-X_{j-1}\right)-\sin \left(X_{j+1}-X_{j-1}\right)-\sin \left(X_{j}-X_{j+1}\right)$.

Suppose the set of nodes $\left\{X_{j}\right\}$ such, that $h=X_{j+1}-X_{j}$, $X_{j}-X_{j-1}=A h, A>0$, and let $x=X_{j}+t h, t \in[0,1]$. Now the solution of system (2) can be written in the form:

$$
w_{j}\left(X_{j}+t h\right)=\frac{\sin (t h-h)+\sin (h+A h)-\sin (A h+t h)}{-\sin (A h)+\sin (h+A h)-\sin (h)},
$$

*e-mail: i.g.burova@spbu.ru 


$$
\begin{gathered}
w_{j+1}\left(X_{j}+t h\right)=\frac{-(\sin (t h)+\sin (A h)-\sin (A h+t h))}{(-\sin (A h)+\sin (h+A h)-\sin (h))}, \\
w_{j-1}\left(X_{j}+t h\right)=\frac{(\sin (t h)-\sin (h)-\sin (-h+t h))}{(-\sin (A h)+\sin (h+A h)-\sin (h))} .
\end{gathered}
$$

It is not difficult to see that the solution of system (2) also can be written as follows:

$$
\begin{aligned}
w_{j}(x) & =\frac{\sin \left(x / 2-X_{j-1} / 2\right) \sin \left(X_{j+1} / 2-x / 2\right)}{\sin \left(X_{j} / 2-X_{j-1} / 2\right) \sin \left(X_{j+1} / 2-X_{j} / 2\right)}, \\
w_{j+1}(x) & =\frac{\sin \left(x / 2-X_{j-1} / 2\right) \sin \left(-X_{j} / 2+x / 2\right)}{\sin \left(X_{j+1} / 2-X_{j-1} / 2\right) \sin \left(X_{j+1} / 2-X_{j} / 2\right)}, \\
w_{j-1}(x) & =\frac{\sin \left(x / 2-X_{j} / 2\right) \sin \left(+x / 2-X_{j+1} / 2\right)}{\sin \left(X_{j-1} / 2-X_{j} / 2\right) \sin \left(X_{j-1} / 2-X_{j+1} / 2\right)} .
\end{aligned}
$$

Under the assumption $h=X_{j+1}-X_{j}, X_{j}-X_{j-1}=A h$, $A>0, x=X_{j}+t h, t \in[0,1]$, the basic splines $w_{j-1}(x)$, $w_{j}(x), w_{j+1}(x), x \in\left[X_{j}, X_{j+1}\right]$, can be written now also as follows:

$$
\begin{gathered}
w_{j}\left(X_{j}+t h\right)=\frac{-\sin (t h / 2+A h / 2) \sin (-h / 2+t h / 2)}{\sin (A h / 2) / \sin (h / 2)}, \\
w_{j+1}\left(X_{j}+t h\right)=\frac{\sin (t h / 2+A h / 2) \sin (t h / 2)}{\sin (A h / 2+h / 2) / \sin (h / 2)} \\
w_{j-1}\left(X_{j}+t h\right)=\frac{\sin (t h / 2) \sin (-h / 2+t h / 2)}{\sin (A h / 2) / \sin (A h / 2+h / 2)} .
\end{gathered}
$$

The last form of the basic splines will be used to construct the approximation.

\subsection{Comparison with polynomial splines}

As it is was shown in paper [6] we can use the following approximation:

$$
G(x)=f\left(X_{j-1}\right) \omega_{j-1}(x)+f\left(X_{j}\right) \omega_{j}(x)+f\left(X_{j+1}\right) \omega_{j+1}(x),
$$

$x \in\left[X_{j}, X_{j+1}\right]$. This approximation will be a polynomial one if we obtain basic functions $\omega_{j-1}(x), \omega_{j}(x), \omega_{j+1}(x)$, $x \in\left[X_{j}, X_{j+1}\right]$, solving the following system:

$$
\begin{gathered}
X_{j-1}^{2} \omega_{j-1}(x)+X_{j}^{2} \omega_{j}(x)+X_{j+1}^{2} \omega_{j+1}(x)=x^{2}, \\
X_{j-1} \omega_{j-1}(x)+X_{j} \omega_{j}(x)+X_{j+1} \omega_{j+1}(x)=x, \\
\omega_{j-1}(x)+\omega_{j}(x)+\omega_{j+1}(x)=1 .
\end{gathered}
$$

Suppose the set of nodes $\left\{X_{j}\right\}$ such, that $h=X_{j+1}-X_{j}=$ $X_{j}-X_{j-1}$. The solution of this system is the following:

$$
\begin{aligned}
\omega_{j}(x) & =\left(x-X_{j-1}\right)\left(X_{j+1}-x\right) / h^{2}, \\
\omega_{j+1}(x) & =\left(x-X_{j}\right)\left(x-X_{j-1}\right) /\left(2 h^{2}\right), \\
\omega_{j-1}(x) & =\left(x-X_{j}\right)\left(x-X_{j+1}\right) /\left(2 h^{2}\right) .
\end{aligned}
$$

Using the notation $x=X_{j}+t h$, we get

$$
\begin{gathered}
\omega_{j}\left(X_{j}+t h\right)=-(t-1)(t+1), \\
\omega_{j+1}\left(X_{j}+t h\right)=t(t+1) / 2, \\
\omega_{j-1}\left(X_{j}+t h\right)=t(t-1) / 2 .
\end{gathered}
$$

Easily it can be shown that there are relations between trigonometrical and polynomial splines:

$$
\begin{aligned}
& w_{j}\left(X_{j}+t h\right)=\omega_{j}\left(X_{j}+t h\right)+O\left(h^{2}\right), \\
& w_{j+1}\left(X_{j}+t h\right)=\omega_{j+1}\left(X_{j}+t h\right)+O\left(h^{2}\right), \\
& w_{j-1}\left(X_{j}+t h\right)=\omega_{j-1}\left(X_{j}+t h\right)+O\left(h^{2}\right) .
\end{aligned}
$$

Theorem 1. Let function $f(x)$ be such that $f \in$ $C^{3}[\alpha, \beta],[\alpha, \beta] \subset[a, b]$. The set of nodes such that $X_{j+1}-X_{j}=X_{j}-X_{j-1}=h$. Then for $x \in\left[X_{j}, X_{j+1}\right]$ we have

$$
\begin{gathered}
|f(x)-G(x)| \leq K_{1} h^{3}\left\|f^{\prime \prime \prime}\right\|_{\left[X_{j-1}, X_{j+1}\right]}, \\
|f(x)-F(x)| \leq K_{2} h^{3}\left\|f^{\prime \prime \prime}+f^{\prime}\right\|_{\left[X_{j-1}, X_{j+1}\right]},
\end{gathered}
$$

where $K_{1}=0.385 / 3$ ! $\approx 0.0642, K_{2}=0.0713$.

Proof. Using the results from the paper [7] we get

$$
\begin{gathered}
f(x)=2 \int_{X_{j}}^{x}\left(f^{\prime \prime \prime}(t)+f^{\prime}(t)\right)(\sin (x / 2-t / 2))^{2} d t+ \\
c_{1}+c_{2} \sin (x)+c_{3} \cos (x),
\end{gathered}
$$

where $c_{i}, i=1,2,3$ are some arbitrary constants. Using expression (1), the solution of the system (2) and expression (7) we receive the estimation (6) of error of approximation with the trigonometrical splines. Using expression (1), the solution of the system (4) and the Taylor series for $f(x)$ we receive the the estimation (5) of error of approximation with the polynomial splines.

Remark. There are examples that show that the constants $K_{1}$ and $K_{2}$ can't be reduced. They are the following: $f_{1}(x)=x^{3} / 6$ for the polynomial splines and $f_{2}(x)=\sin (x)-\cos (x)+x$ for the trigonometrical splines. Let us take $h=1, X_{j}=0, X_{j+1}=1, X_{j-1}=-1$ and construct the trigonometrical approximation $F(x)$ and polynomial approximation $G(x)$ using (1).

We have for these functions:

$$
f_{1}^{\prime \prime \prime}(x)=1, f_{2}^{\prime \prime \prime}(x)+f_{2}^{\prime}(x)=1,
$$

so at point $x=0.5708$ we receive $F(x)-f_{2}(x)=0.0713$, and at point $x=0.57735$ we receive $G(x)-f_{1}(x)=0.0642$.

The results of the application the trigonometrical splines for the approximation of functions are given in table 1. The results of application the polynomial splines for the approximation of the same functions are given in table 2 . In the second column of table 1 and table 2 the maximums of the practical errors of approximations in absolute values are done. In the third column of table 1 and table 2 the maximums of the theoretical errors of approximations in absolute values are done. Calculations for the both tables were made in Maple with Digits $=15,[a, b]=[-1,1]$, $h=0.1$.

\section{Approximation with right splines}

Now we consider the approximation of a function $f(x)$, $x \in\left[X_{j}, X_{j+1}\right]$, with right trigonometric splines. We can construct an approximation of function $f(x)$, also in the form:

$$
F(x)=f\left(X_{j}\right) w_{j}(x)+f\left(X_{j+1}\right) w_{j+1}(x)+f\left(X_{j+2}\right) w_{j+2}(x) .
$$


Table 1. The errors of approximation with the trigonometrical splines

\begin{tabular}{lll}
\hline$f(x)$ & Prac.err. & Theor.err. \\
\hline $\sin (3 x)$ & $0.153 \mathrm{e}-2$ & $0.171 \mathrm{e}-2$ \\
$1 /\left(1+25 x^{2}\right)$ & $0.294 \mathrm{e}-1$ & $0.413 \mathrm{e}-1$ \\
$x^{3}$ & $0.573 \mathrm{e}-3$ & $0.642 \mathrm{e}-3$ \\
$\sin (x)-\cos (x)+x$ & $0.642 \mathrm{e}-4$ & $0.713 \mathrm{e}-4$ \\
\hline
\end{tabular}

Table 2. The errors of approximation with the polynomial splines

\begin{tabular}{lll}
\hline$f(x)$ & Prac.err. & Theor.err. \\
\hline $\sin (3 x)$ & $0.1721 \mathrm{e}-2$ & $0.1732 \mathrm{e}-2$ \\
$1 /\left(1+25 x^{2}\right)$ & $0.2957 \mathrm{e}-1$ & $0.3754 \mathrm{e}-1$ \\
$x^{3}$ & $0.3849 \mathrm{e}-3$ & $0.3850 \mathrm{e}-3$ \\
$\sin (x)-\cos (x)+x$ & $0.9061 \mathrm{e}-4$ & $0.9074 \mathrm{e}-4$ \\
\hline
\end{tabular}

We obtain basic functions $w_{j}(x), w_{j+1}(x), w_{j+2}(x)$ from the following system:

$\sin \left(X_{j}\right) W_{j}(x)+\sin \left(X_{j+1}\right) W_{j+1}(x)+\sin \left(X_{j+2}\right) W_{j+2}(x)=\sin (x)$, $\cos \left(X_{j}\right) W_{j}(x)+\cos \left(X_{j+1}\right) W_{j+1}(x)+\cos \left(X_{j+2}\right) W_{j+2}(x)=\cos (x)$,

$$
W_{j}(x)+W_{j+1}(x)+W_{j+2}(x)=1 .
$$

The solution of this system can be written as follows:

$W_{j}(x)=\left(-\sin \left(X_{j+1}-X_{j+2}\right)-\sin \left(X_{j+2}-x\right)+\sin \left(X_{j+1}-x\right)\right) / S_{j}$,

$W_{j+1}(x)=\left(-\sin \left(-x+X_{j}\right)+\sin \left(X_{j}-X_{j+2}\right)+\sin \left(X_{j+2}-x\right)\right) / S_{j}$

$W_{j+2}(x)=\left(\sin \left(-x+X_{j}\right)-\sin \left(-X_{j+1}+X_{j}\right)-\sin \left(X_{j+1}-x\right)\right) / S_{j}$,

where

$$
S_{j}=\left(\sin \left(X_{j}-X_{j+2}\right)-\sin \left(-X_{j+1}+X_{j}\right)-\sin \left(X_{j+1}-X_{j+2}\right)\right) .
$$

\section{Interval extention}

Theorem 1 helps us to choose the correct length $h=X_{j+1}-$ $X_{j}$ of the interval $\left[X_{j}, X_{j+1}\right]$.

Suppose we know the values of function $f(x)$ at nodes $\left\{X_{k}\right\}$. Using formulas (1), (3) or (8) with trigonometrical splines and the technique of interval analysis [4] we can construct the upper and lower boundaries for every interval $Y=\left[X_{j}, X_{j+1}\right]$. Thus we avoid the calculations of approximation $f(x)$ in many points of every interval $\left[X_{j}, X_{j+1}\right]$ if we need to know the boundaries of the interval, where the function $f$ varieties. In order to obtain the boundaries of variety $f(x)$ we construct the approximation $F(x), x \in Y$ in form (1) and consider $F(Y)$.

In order to get the narrowest estimation interval we transform formulas (3). First, we consider the estimate of the lower bound of the estimating interval of the basis spline $w_{j-1}(x)$.

Let $X_{j-1}^{\max }$ be the maximum

$X_{j-1}^{\max }=\max _{x \in\left[X_{j}, X_{j+1}\right]}\left(\cos \left(X_{j} / 2-x+X_{j+1} / 2\right)\right.$.

Then the upper boundary of $w_{j-1}$ will be the following $w_{j-1}^{M A}=2 \sin \left(X_{j} / 2-X_{j+1} / 2\right) /\left(\sin \left(X_{j}-X_{j-1}\right)-\sin \left(X_{j+1}-\right.\right.$ $\left.\left.X_{j-1}\right)-\sin \left(-X_{j+1}+X_{j}\right)\right) \cdot X_{j-1}^{\max }+w_{j-1}^{A}$, where
$w_{j-1}^{A}=\sin \left(X_{j+1}-X_{j}\right) /\left(\sin \left(X_{j}-X_{j-1}\right)-\sin \left(X_{j+1}-X_{j-1}\right)-\right.$ $\left.\sin \left(-X_{j+1}+X_{j}\right)\right)$.

After calculating the upper boundaries of $w_{j-1}, w_{j}$ and $w_{j+1}$ we can calculate the upper boundary of $F(x)$. Now the upper boundary of $F(x)$ will be the following:

$$
F^{M A X}=f\left(X_{j-1}\right) w_{j-1}^{M A}+f\left(X_{j}\right) w_{j}^{M A}+f\left(X_{j+1}\right) w_{j+1}^{M A} .
$$

The results of the interval extension of function $f(x)$ are given in figures 1-4 and 7-8. The plots of function $f(x)$ and its upper and lower boundaries are given in figure 1 when $f(x)=\sin (x)$ and in figure 2 when $f(x)=\cos (x)$.

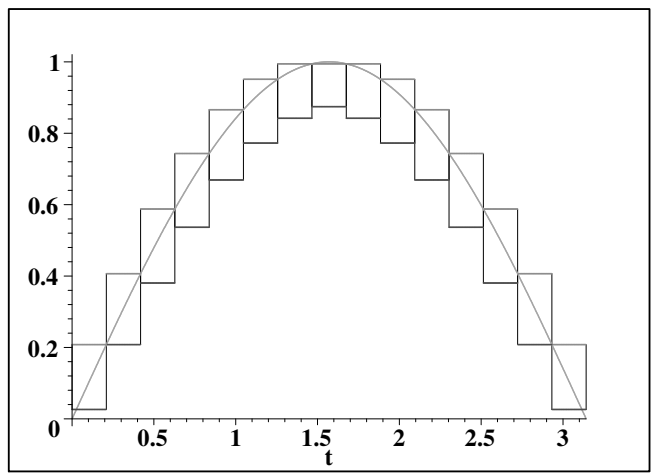

Figure 1. The plots of function $f(x)=\sin (x)$ and its upper and lower boundaries.

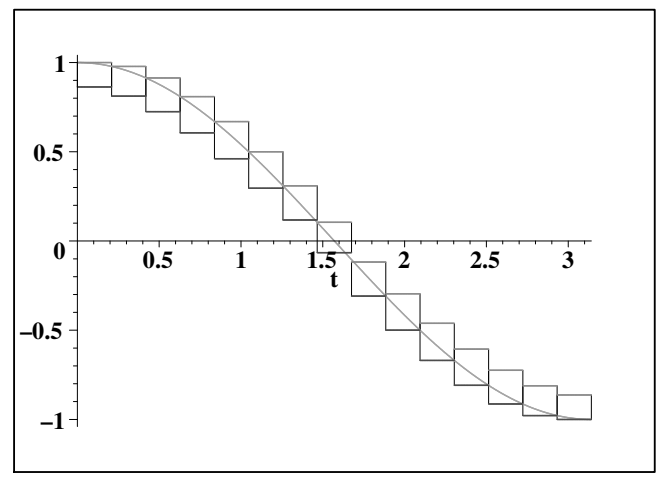

Figure 2. The plots of function $f(x)=\cos (x)$ and its upper and lower boundaries.

The plots of function $1 /\left(1+25 x^{2}\right)$ and its upper and lower boundaries are given in figure 3 : on $[0, \pi]$ figure 3 .

The plots of function $\sin (x)-\cos (x)+x$ and its upper and lower boundaries are given in figure 4 : on $[0, \pi]$ figure 4

Suppose we know the values function $\sin (7 x)-\cos (9 x)$ in points $X_{j}=j \cdot \pi / 15, j=0,1, \ldots, 15$. The information about the function can be represented in more then one possibility. At first, we can draw the pointplot of the points. The plot of points $\sin \left(7 X_{j}\right)-\cos \left(9 X_{j}\right), X_{j}=j \cdot \pi / 15$, $j=0,1, \ldots, 15$ on $[0, \pi]$ is given in figure 5 .

After this is done, we could connect the points with lines. This plot is given in figure 6. But instead of the last one we could use the presentation of the function with our method of interval extension on every set interval. The result is given in figure 6 . 


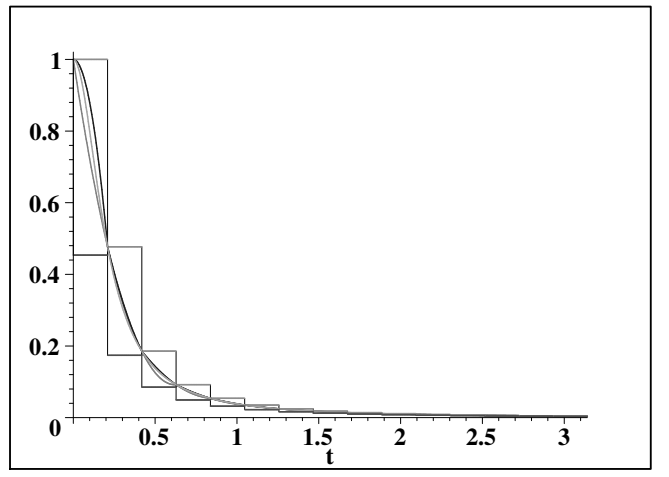

Figure 3. The plots of function $1 /\left(1+25 x^{2}\right)$ and its upper and lower boundaries: on $[0, \pi]$

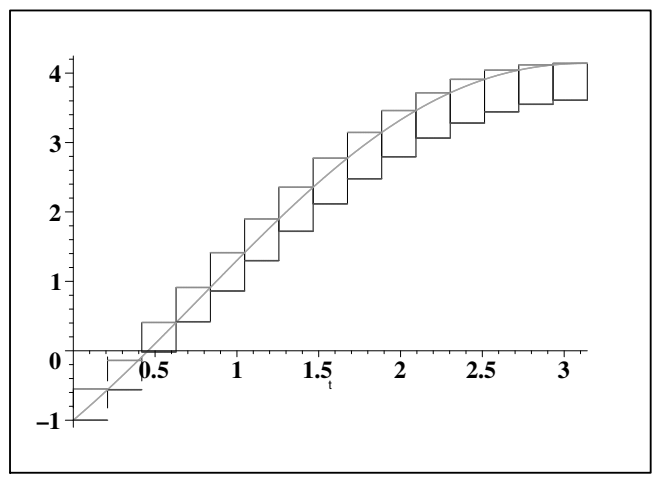

Figure 4. The plots of function $\sin (x)-\cos (x)+x$ and upper and lower boundaries: on $[0, \pi]$

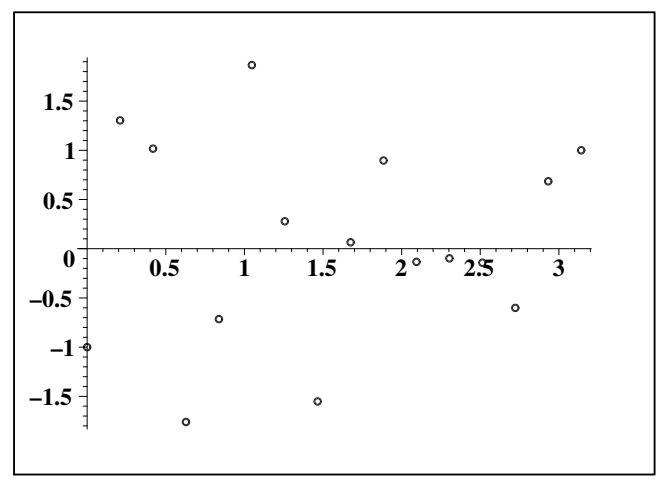

Figure 5. The plot of points $\sin \left(7 X_{j}\right)-\cos \left(9 X_{j}\right), X_{j}=j \cdot \pi / 15$, $j=0,1, \ldots, 15$ on $[0, \pi]$

The plots of the upper and lower boundaries of the function $\sin (7 x)-\cos (9 x)$ and points $\sin \left(7 X_{j}\right)-\cos \left(9 X_{j}\right)$, $X_{j}=j \cdot \pi / 15, j=0,1, \ldots, 15$ on $[0, \pi]$ are given in figure 7 .

The plots of the upper and lower boundaries of the function $\sin (7 x)-\cos (9 x)$, points $\sin \left(7 X_{j}\right)-\cos \left(9 X_{j}\right)$, $X_{j}=j \cdot \pi / 15, j=0,1, \ldots, 15$, and lines, which connects the points on $[0, \pi]$ are given on figure 8 .

The plots of function $\sin (7 x)-\cos (9 x)$, the upper and lower boundaries of the function $\sin (7 x)-\cos (9 x)$, points $\sin \left(7 X_{j}\right)-\cos \left(9 X_{j}\right), X_{j}=j \cdot \pi / 15, j=0,1, \ldots, 15$ on $[0, \pi]$ are given on figure 9 .

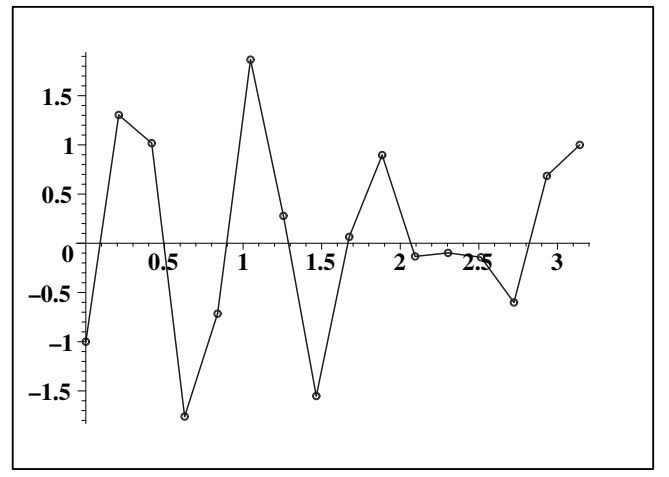

Figure 6. The plots of points $\sin \left(7 X_{j}\right)-\cos \left(9 X_{j}\right), X_{j}=j \cdot \pi / 15$, $j=0,1, \ldots, 15$ and lines, which connects the points on $[0, \pi]$

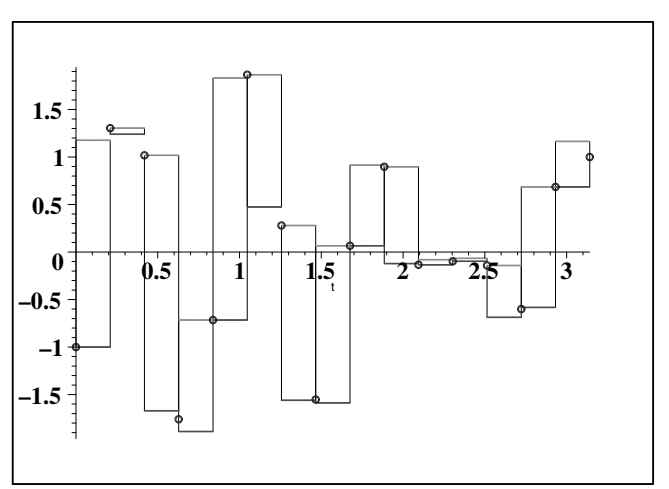

Figure 7. The plots of the upper and lower boundaries of the function $\sin (7 x)-\cos (9 x)$ and points $\sin \left(7 X_{j}\right)-\cos \left(9 X_{j}\right), X_{j}=$ $j \cdot \pi / 15, j=0,1, \ldots, 15$ on $[0, \pi]$

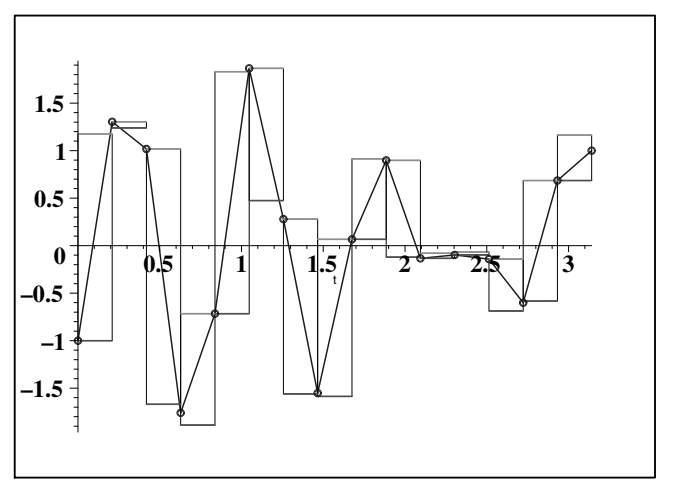

Figure 8. The plots of the upper and lower boundaries of the function $\sin (7 x)-\cos (9 x)$, points $\sin \left(7 X_{j}\right)-\cos \left(9 X_{j}\right), X_{j}=j$. $\pi / 15, j=0,1, \ldots, 15$, and lines, which connects the points on $[0, \pi]$

The plots of function $\sin (7 x)-\cos (9 x)$ and its approximation (thick line) with the left splines on $[0, \pi]$ are given on figure 10.

The plot of the error of approximation of the function $\sin (7 x)-\cos (9 x)$ with the left trigonometrical splines on $[0, \pi]$ is given on figure 11 . The practical error of approximation is the following: $\max _{[0, \pi]}|f(x)-F(x)|=0.52$. 


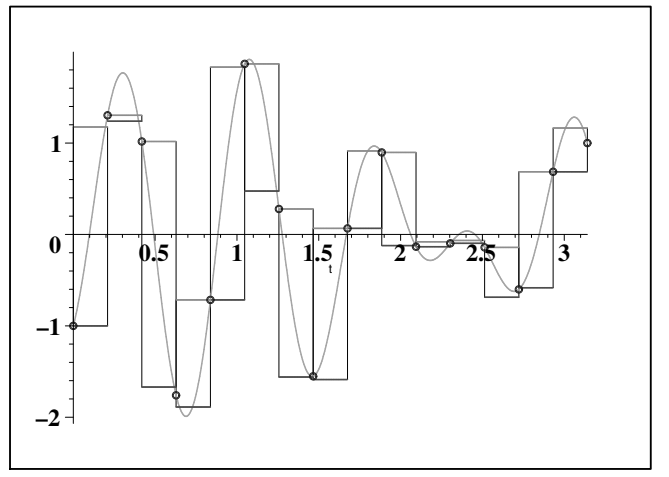

Figure 9. The plots of function $\sin (7 x)-\cos (9 x)$, the upper and lower boundaries of the function $\sin (7 x)-\cos (9 x)$, points $\sin \left(7 X_{j}\right)-\cos \left(9 X_{j}\right), X_{j}=j \cdot \pi / 15, j=0,1, \ldots, 15$ on $[0, \pi]$

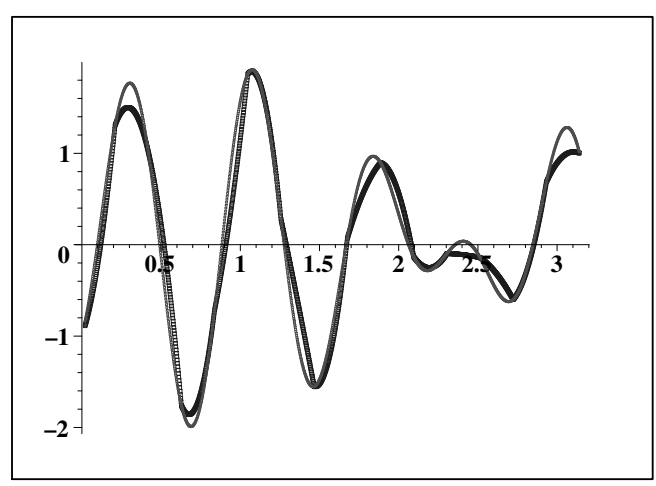

Figure 10. The plots of function $\sin (7 x)-\cos (9 x)$ and its approximation (thick line) with the left splines on $[0, \pi]$

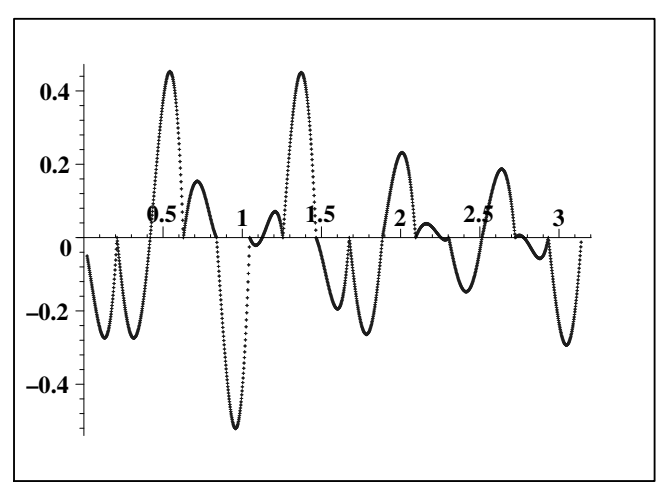

Figure 11. The plot of the error of approximation of the function $\sin (7 x)-\cos (9 x)$ with the left splines on $[0, \pi]$

\section{Conclusion}

In this paper we calculate the constants that can't be reduced in the theorem of approximation with trigonometrical splines and present the results of working the program of constructing interval extension. The results show that in many cases connected with approximation trigonometrical functions application approximations with trigonometrical splines gives better results then approximation with polynomial splines. To avoid calculation in many points we can use interval extension if we need to know only the upper and the lower boundaries of variation of the function. But we have to hold in mind the theorem of approximation.

\section{References}

[1] D.Michel, A.Zidna, Interval-krawczyk approach for solving nonlinear equations systems in B-spline form, Advances in Intelligent Systems and Computing, 359, pp. 455-465 (2015)

[2] X.Liu, A framework of verified eigenvalue bounds for self-adjoint differential operators, Applied Mathematics and Computation, 267, pp. 341-355 (2015)

[3] L.Xie, J. Liu, J. Zhang, X.Man, Evidence-TheoryBased Analysis for Structural-Acoustic Field with Epistemic Uncertainties, International Journal of Computational Methods, 14 (2) (2017)

[4] G.Alefeld, J.Herzberger, Introduction to Interval Computations (Academic Press, New York, 1983), $360 \mathrm{p}$.

[5] I.G.Burova, A.A.Vartanova, Interval Estimation of Polynomial Splines of the Fifth Order, Proceedings 2017 4th International Conference on Mathematics and Computers in Sciences and in Industry, MCSI 2017, pp. 293-297 (2018-January)

[6] I.G.Burova, Construction of trigonometric splines, Vestnik St. Petersburg University: Mathematics, 37 (2), pp. 6-11 (2004)

[7] I.G.Burova, On left integro-differential splines and Cauchy problem, International Journal of Mathematical Models and Methods in Applied Sciences, 9, pp. 683-690 (2015) 